\title{
ON THE OPTIMAL WEIGHT FUNCTION IN THE GOLDSTON-PINTZ-YILDIRIM METHOD FOR FINDING SMALL GAPS BETWEEN CONSECUTIVE PRIMES
}

\author{
BÁLINT FARKAS, JÁNOS PINTZ, AND SZILÁRD RÉVÉSZ
}

\begin{abstract}
We work out the optimization problem, initiated by K. Soundararajan, for the choice of the underlying polynomial $P$ used in the construction of the weight function in the Goldston-Pintz-Ylldırım method for finding small gaps between primes. First we reformulate to a maximization problem on $L^{2}[0,1]$ for a self-adjoint operator $T$, the norm of which is then the maximal eigenvalue of $T$. To find eigenfunctions and eigenvalues, we derive a differential equation which can be explicitly solved. The aimed maximal value is $S(k)=4 /\left(k+c k^{1 / 3}\right)$, achieved by the $k-1^{\text {st }}$ integral of $x^{1-k / 2} J_{k-2}\left(\alpha_{1} \sqrt{x}\right)$, where $\alpha_{1} \sim c k^{1 / 3}$ is the first positive root of the $k-2^{\text {nd }}$ Bessel function $J_{k-2}$. As this naturally gives rise to a number of technical problems in the application of the GPY method, we also construct a polynomial $P$ which is a simpler function yet it furnishes an approximately optimal extremal quantity, $4 /\left(k+C k^{1 / 3}\right)$ with some other constant $C$. In the forthcoming paper of J. Pintz 8$]$ it is indeed shown how this quasi-optimal choice of the polynomial in the weight finally can exploit the GPY method to its theoretical limits.
\end{abstract}

\section{INTRODUCTION}

1.1. The extremal problem as given by Soundararajan. In his work [11] Soundararajan presents and analyzes the proof of Goldston-Pintz-Yıldırım yielding small gaps between primes. Among others he raises and answers one of the most important problems of the field: Is it possible to modify the weight function $a(n)$ in such a way that the method would lead to infinitely many bounded gaps between consecutive primes. If we consider the weight functions in full generality, that is all functions $a(n)$, then this leads essentially to a tautology. For example, defining $a(n)=1$ if both $n$ and $n+2$ are primes, and otherwise setting $a(n)=0$, the summatory function of $a(n)$ describes the number of twin primes up to $x$. Thus we cannot hope an asymptotic evaluation of the summatory function. We briefly describe the feasible choices of the weight function $a$. Let us take an admissible $k$-tuple $\mathcal{H}=\left\{h_{1}, \ldots, h_{k}\right\}$ meaning that there is no prime $p$ with the property that the elements $h_{i}$ of $\mathcal{H}$ cover all residue classes $\bmod p$. Let $P_{\mathcal{H}}(n)=\prod_{i=1}^{k}\left(n+h_{i}\right)$ and let us define $\lambda_{d}=\mu(d) P\left(\frac{\log (R / d)}{\log R}\right)$ with a nice function $P$, for example a polynomial, with the additional property $\lambda_{1}=1$, which is equivalent to $P(1)=1$. Afterwards we reduce our choice of $a(n)$ to those of type $a(n)=\sum_{d \leq R, d \mid P_{\mathcal{H}}(n)} \lambda_{d}$ and try to evaluate the summatory function of $a(n)$ and that of $a(n) \chi(n+\bar{h})$, where $h$ is an arbitrary number

\footnotetext{
Date: June 11, 2013.

2000 Mathematics Subject Classification. Primary 11N05, 47A75. Secondary 47A53, 49J05, 49K15, $49 \mathrm{~N} 10$.

Key words and phrases. Prime distribution, gaps between primes, Goldston-Pintz-Ylldırım method, selfadjoint Fredholm type operator, Bessel differential equation, Bessel functions of the first and second kind.

Supported in part by the Hungarian National Foundation for Scientific Research, Project \# K-81658, K-100291, K-100461 and NK-104183. Work done in the framework of the project ERC-AdG 228005.
} 
with $h<\log n$ and $\chi$ is the characteristic function of the primes. (In case of bounded gaps between primes it is sufficient to consider the case when $h=h_{i}, i=1,2, \ldots, k$.)

Soundararajan explains, how the optimal weight function $a(n)$, hence $\lambda_{d}$, should be chosen to obtain best result: see formula (8) in [11. In order to get this optimum, he also explains the choice $\lambda_{d}:=\mu(d) P\left(\frac{\log (R / d)}{\log R}\right)$ where $P$ is some suitably nice function, like a polynomial or at least a sufficiently many times (at least $k$ times) differentiable, smooth function on $[0,1]$ (or at least on $[0,1)$ ), vanishing at least in the order $k$ at 0 , and satisfying the normalization $P(1)=1$. Then, according to the analysis by Soundararajan, the optimal choice for $a(n)$ and $\lambda_{d}$ is equivalent to looking for the maximal possible value of (12) of [11], i.e., to determining

(1)

$$
S(k):=\sup _{P}\left(\int_{0}^{1} \frac{x^{k-2}}{(k-2) !}\left(P^{(k-1)}(1-x)\right)^{2} d x\right) /\left(\int_{0}^{1} \frac{x^{k-1}}{(k-1) !}\left(P^{(k)}(1-x)\right)^{2} d x\right),
$$

where the set of functions $P$, to be taken into account in the supremum, can be the set of certain polynomials as before, or more generally a family of functions subject to some conditions.

Soundararajan [11] shows that the question whether we are able to find in this way infinitely many bounded gaps between primes is equivalent to the problem whether there exists any natural number $k$ with $S(k)>4 / k$. Then he mentions that the opposite inequality $S(k)<4 / k$ holds for all $k$ and therefore the method cannot yield infinitely many bounded prime gaps. (In an earlier unpublished note [10] he gives the short proof of this fact; we will reproduce this in $\$ 2.3$. His considerations also lead easily to the stronger inequality $S(k)<4 /(k+c \log k)$, cf. $\$ 2.3)$. Although his work answered negatively the above mentioned central problem, it gave some hints but did not answer the question: What is the best weight function that can be chosen, and what size of gaps are implied by it? In their work [5] Goldston, Pintz and Yıldırım showed that if one takes $P(x)=x^{k+\ell}$, where $k$ and $\ell$ are allowed to tend to infinity with the size $N$ of the primes considered, then with several essential modifications of the original method one can reach infinitely many prime gaps of size essentially $\sqrt{\log p}$. (To have an idea of the difficulties it is enough to mention that the rather condensed proof of the result needs about 40 additional pages beyond the original one, presented with many details and explanations in [4]. However, a shortened, simplified and more condensed version [6 needs only 5 pages). In this case $\ell=c \sqrt{k}$ and the value of the fraction (11) is $4 /\left(k+c^{\prime} \sqrt{k}\right)$ for the given choice of $P(x)=x^{k+\ell}$. Beyond the mentioned important fact that $k$ and $\ell$ are unbounded in [5], the scheme of the proof is similar but not the same as in the simplified version of Soundararajan [11]. However, a careful analysis suggests that in order to find the limits of the method it is necessary (but as discussed a little later, not necessarily sufficient) to find the size of $S(k)$ as $k$ tends to infinity together with the function $P$ which yields a maximum (if it exists) in the supremum, or at least a function $P$ which yields a value "enough close" to the supremum.

1.2. Conditions and normalizations. Before proceeding, let us discuss right here the issue of conditions and normalizations in the formulation of this maximization problem. First, it is clear that $P^{(k)}$ remains unchanged, if we add any constant to $P^{(k-1)}$. Thus the extremal problem becomes unbounded under addition of a free constant, hence at least some conditions must certainly control this divergence. 
In the number theory construction of Goldston-Pintz-Yıldırım, (by now generally abbreviated as the "GPY method") the natural restriction is that $P$ must be a polynomial divisible by $x^{k}$-or, if we try to generalize the method, then a $k$-times continuously differentiable function with $P^{(j)}$ vanishing at 0 for $j=0, \ldots, k-1$. That is $P(x)=\frac{P^{(k)}(0)}{k !} x^{k}+o\left(x^{k+1}\right)$. The reason for that is the fact that the whole idea hinges upon the use of the generalized Möbius inversion, more precisely of the $\Lambda_{j}$ function, which must be zero for numbers having at least $k$ prime factors - always satisfied by the numbers represented by the product form $\left(n+h_{1}\right) \cdots\left(n+h_{k}\right)$ in the construction. So for any meaningful weight function we need to use weights not containing any smaller power $x^{j}$ than $x^{k}$. In other words, we should assume here $P$ having a zero of order $k$, i.e., $P(0)=P^{\prime}(0)=\cdots=P^{(k-1)}(0)=0$, while $P^{(k)}(0)$ can be arbitrary.

The analysis of Soundararajan exposed the question, whether a linear combination of monomials, i.e., a polynomial, or perhaps some more sophisticated choice of a weight function, may perhaps improve even upon this. We can say that the theoretical limit of the GPY method is the result, obtainable in principle by a choice of the weight function $P$ maximizing the extremal quantity (1). Yet it is to be noted that the technicalities of GPY are far more substantial than to simply "substituting any $P$ " in it would automatically lead to a result - it is not even that clear, what result would follow from a given weight function. Therefore, to test the limits of the GPY method, we should break our approach into two parts. First, we look for the optimization of the weight $P$, in the sense of (1), and second, we extend the GPY method using that weight function. This paper is concerned with this first question, and the second part of this program is carried out in [8].

The aim of the present analysis is to settle the issue of optimization in Problem (1). We find the optimal order, and the maximizer of the problem (1), furthermore, as this maximum can be achieved by a relatively sophisticated choice of the weight function $P$ actually a transformed Bessel function - we also construct a polynomial weight which is approximately optimal in (1).

Part of these results were reached by J. B. Conrey and his colleagues at the American Institute of Mathematics already in 2005. Using a calculus of variation argument they found the Bessel function $J_{k-2}$ and made some calculations for concrete values of $k$ (without analyzing the case $k \rightarrow \infty$ ). The fact that the Bessel functions may perform better than polynomials in the GPY method is also briefly noted in the book of J. B. Friedlander and H. Iwaniec [3] without going into details.

\subsection{Structure of the paper. In this paper we proceed along the following course.}

Interpreting the problem in the widest possible function class which makes sense (i.e., when at least the occurring integrals exist finitely) in Section 3 we make several further reformulations until we arrive at a maximization problem in the Hilbert space $L^{2}[0,1]$. Exploiting the rich structure of Hilbert spaces, and the particular properties of the reformulation as a certain quadratic form with a Fredholm-type operator, we derive existence of maximizing functions in this wide function class. Then we also exploit the concrete form of the kernel in our Fredholm-type operator and compute that the maximizers, or, more generally, eigenfunctions, are necessarily smooth. Furthermore, in Section 4 we find that these eigenfunctions satisfy certain differential equations. The solutions are then found to be transformed variants of certain Bessel functions. Also it turns out that the solutions are analytic, and they yield a function value in the extremal problem directly related to the choice of a parameter, which, due to the initial value restriction $P^{(k-1)}(0)=0$, must be a zero of the arising Bessel function $J_{k-2}$. Finally these combine to the full description 
of the maximal value $S(k)$ together with the precise form of the extremal function. From the well-known asymptotic formula for the first zero of the Bessel function $J_{m}$, when $m \rightarrow \infty$, we derive that $S(k)$ is precisely asymptotic to $\frac{4}{k+c k^{1 / 3}}$ with a concretely known constant $c=3.7115 \ldots$

Unfortunately, in spite of analyticity and power series expansion, the found extremal function is too complicated to be used in the number theory method of GPY. Basically, we need restrictions on the degree and the coefficient size for the powers appearing in the weight function $P$ to make the complicated method work in a technically feasible way. As discussed in Section 5, not even calculations using the power series expansion of Bessel functions lead to feasible expressions. Therefore, finally we look for quasi-optimal polynomials, which still achieve close to extremal values. The result of the last section is the concrete construction of a polynomial $P$ satisfying the needed technical requirements and still achieving in (1) a ratio of the order $\frac{4}{k+C k^{1 / 3}}$ with some other constant $C$. That suffices in the method of GPY, because the value of the constant $C$ does not increase the order, only the arising constants, in the final result.

Settling the issue of the search of optimal and quasi-optimal weights, the door opens up for revisiting the method of GPY and not only improve upon all the known results, but also push the available techniques to the theoretical limits of that method. This closely connected work is carried out in the paper [8].

\section{Reformulations and the Finiteness of $S(k)$}

2.1. Reformulations. The normalization $P(1)=1$ is rather inconvenient because the next reformulation (still following Soundararajan) is to put $Q(y):=P^{(k-1)}(y)$, a completely logical step in view of the fact that no values of $P, P^{\prime}$, etc. $P^{(k-2)}$ occur in the actual optimization problem (11) and that the still occurring $P^{(k-1)}$ and $P^{(k)}$ can be nicely expressed as $Q$ and $Q^{\prime}$. So in line with the restriction that $P$ vanishes at least to the order $k$ at 0 , following Soundararajan we write

$$
\begin{aligned}
P(x) & =\int_{0}^{x} \int_{0}^{x_{1}} \ldots \int_{0}^{x_{k-2}} P^{(k-1)}\left(x_{k-1}\right) d x_{k-1} \ldots d x_{1}=\int_{0}^{x} \int_{0}^{x_{1}} \ldots \int_{0}^{x_{k-1}} P^{(k)}\left(x_{k}\right) d x_{k-1} \ldots d x_{1} \\
& =\int_{0}^{x} \int_{0}^{x_{1}} \ldots \int_{0}^{x_{k-2}} Q\left(x_{k-1}\right) d x_{k-1} \ldots d x_{1}=\int_{0}^{x} \int_{0}^{x_{1}} \ldots \int_{0}^{x_{k-1}} Q^{\prime}\left(x_{k}\right) d x_{k} \ldots d x_{1} .
\end{aligned}
$$

Therefore, $P(0)=\cdots=P^{(k-2)}(0)=P^{(k-1)}(0)=0$ transforms to the simpler requirement that $Q(0)=0$, while the corresponding $P$ is obtained by the above integrals directly. Let us record one more thing here: The condition that $P(1)=1$, expressed in terms of $Q$, is a linear restriction, as $I(Q):=(P(1)=) \int_{0}^{1} \int_{0}^{x_{1}} \ldots \int_{0}^{x_{k-2}} Q\left(x_{k-1}\right) d x_{k-1} \ldots d x_{1}$ is just a linear functional on the function $Q$. To express it in a more condensed, closed form, we may apply Fubini's theorem to get a representation in the form of the well-known Liouville integral

$$
\begin{aligned}
& P(x)=\int_{0}^{x} Q(t) \frac{(x-t)^{k-2}}{(k-2) !} d t \\
& P(1)=\int_{0}^{1} Q(t) \frac{(1-t)^{k-2}}{(k-2) !} d t=\int_{0}^{1} Q(1-y) \frac{y^{k-2}}{(k-2) !} d t .
\end{aligned}
$$

Note the similarity to the numerator of the quotient in (1). It is thus immediate by the Cauchy-Schwarz inequality that $P(1)$ is a finite, convergent integral whenever the 
Lebesgue integral $\int_{0}^{1} Q^{2}(1-y) \frac{y^{k-2}}{(k-2) !} d y$ exists. That is, no special requirement is needed to this effect once we guarantee that the numerator and denominator in (1) are well-defined.

In all, we were to look for maximum in the family

$$
\mathcal{P}:=\left\{P(x)=\int_{0}^{x} \int_{0}^{x_{1}} \ldots \int_{0}^{x_{k-2}} P^{(k-1)}\left(x_{k-1}\right) d x_{k-1} \ldots d x_{1}, \quad P(1)=1, \quad P^{(k-1)}(0)=0\right\},
$$

but following Soundararajan we changed the setup to

$$
\mathcal{Q}_{1}:=\left\{Q: \int_{0}^{1} Q(1-y) \frac{y^{k-2}}{(k-2) !} d y=1, \quad Q(0)=0\right\},
$$

where now $Q$ can be any (say, continuously differentiable) function satisfying the requirements. This also means that we want the occurring functions to belong to a suitable function class, to be specified later. The quantity we seek to maximize is then expressed as

$$
\frac{\int_{0}^{1} n y^{n-1} Q^{2}(1-y) d y}{\int_{0}^{1} y^{n} Q^{\prime 2}(1-y) d y} \quad(n:=k-1),
$$

which again is a fraction of two expressions, both quadratic homogeneous in $Q$. Therefore, the ratio will be the same for $c Q$ with any $c \neq 0$ and the original question can thus be rewritten as looking for the supremum of these quantities among functions in

$$
\mathcal{Q}^{\star}:=\left\{Q: \int_{0}^{1} y^{k-2} Q(1-y) d y \neq 0, \quad Q(0)=0\right\} .
$$

Continuity of $Q^{\prime}$ is not indispensable, but of course the ratio must be a ratio of finite quantities, with a nonzero and finite denominator, hence we need still to restrict considerations to functions $Q^{\prime} \not \equiv 0$ or, in general allowing discontinuous functions, $Q^{\prime}$ not zero almost everywhere and also satisfying $\int_{0}^{1} x^{n} Q^{\prime 2}(1-x) d x<\infty$.

We will see in a moment-see the proof of the forthcoming Proposition 1-that this latter condition also implies that even $\int n x^{n-1} Q^{2}(1-x) d x<\infty$, as needed. Furthermore, together with the restriction that $Q(0)=0$, we see that $Q$ is constant if only $Q \equiv 0$, so we need to exclude only this obviously singular case. Otherwise also the numerator remans finite, i.e. the ratio (4) exists finitely, whence $S(k)$ exists at least as a supremum of certain finite, positive quantities.

Let us observe that the condition that $P(1) \neq 0$, is a linear condition, equivalently stated in the form that the linear functiona $Q \longrightarrow \int_{0}^{1} Q(1-y) y^{n-1} d y$ on the function space of admissible functions $Q$ should not vanish. In other words, the subset which falls out of consideration for not meeting this condition is the kernel subspace of the linear functional, which is of codimension one-in view of the fact that the functional itself is not identically zero, obvious from looking at functions $Q$ with $\left.Q\right|_{(0,1)}>0$ certainly yielding positive functional values-, so a hyperplane $\mathcal{H}$ of our linear function space $\mathcal{X}$ (whatever choice of the function space and respective norm we make later on).

Therefore, dropping the restriction that $P(1)=Q(0) \neq 0$ means only that $q \notin \mathcal{H}$ is dropped. In the following we will find the supremum on $\mathcal{X}$, and actually will show that here the supremum is finite, attained at certain maximizers.

\footnotetext{
${ }^{1}$ Linearity is clear, once the integral is defined finitely. Then again, finiteness of $\int_{0}^{1} y^{n-1} Q^{2}(1-y) d y$, appearing in the numerator of the extremal quantity, ensures by means of the Cauchy-Schwarz inequality, finiteness of the functional values, too. So for the rest of the argument to be valid, it suffices to check finiteness of the numerator of (4).
} 
The only issue, which may bother us a little, if the actual maximizers will belong to the singular hyperplane $\mathcal{H}$, or stay in $\mathcal{X} \backslash \mathcal{H}$. That we should check at the end. But maximizers $q \in \mathcal{X}$ will actually be positive functions, so the value of the functional $I(q)=P(1)$ will be necessarily positive for these, and maximum over $\mathcal{X}$ or $\mathcal{X} \backslash \mathcal{H}$ will thus be seen to be the same. We will leave it to the reader to check this and from now on pass on to the class $\mathcal{X}$.

2.2. The choice of the function class of the extremal problem (1). In view of the above, let us fix the function class, where the extremal problem (1), initiated by Soundararajan, will be considered, as follows. Write $q(x)=Q(1-x)$ as before. Then the whole problem becomes

(5) $\max (k-1)\left(\int_{0}^{1} x^{k-2} q^{2}(x) d x\right) /\left(\int_{0}^{1} x^{k-1} q^{\prime 2}(x) d x\right) \quad$ under condition $q(1)=0$,

understood in an appropriate function class $\mathcal{X}$, like, e.g., $C^{1}[0,1]$.

Partial integration in the numerator and $q(1)=0$ yields now the reformulation

$$
S(k)=\sup _{q \in \mathcal{X}, q(1)=0, q \neq \mathbf{0}}\left(-2 \int_{0}^{1} x^{k-1} q^{\prime}(x) q(x) d x\right) /\left(\int_{0}^{1} x^{k-1} q^{\prime 2}(x) d x\right) .
$$

Certainly we want the denominator to be finite, so we assume that our function class is chosen in such a way that for any $q \in \mathcal{X}$ this weighted square integral of $q^{\prime}$ converges. This implies the convergence of the numerator (as we'll see soon) and consequently that also the positive, nondegenerate linear functional $I(q):=(k-2) ! P(1)=\int_{0}^{1} q(t) t^{k-2} d t$ is well-defined, finite. So now we fix the largest function space we may deal with as

$$
\mathcal{X}:=\left\{q:(0,1] \rightarrow \mathbb{R}: q(x)=-\int_{x}^{1} q^{\prime}(t) d t, \int_{0}^{1} x^{k-1} q^{\prime 2}(x) d x<\infty\right\} .
$$

The definition above is understood to mean that any $q \in \mathcal{X}$ is an absolutely continuous function on each compact subinterval of $(0,1]$, whence $q^{\prime} \in L_{\mathrm{loc}}^{1}(0,1]$ and $q(x)$ exists as a Lebesgue integral of $q^{\prime}$, and in view of the second condition, $q^{\prime}$ is also square-integrable on $[0,1]$ with respect to the weight $x^{k-1}$.

2.3. An estimation of the extremal value. Before proceeding let us stop for a little further analysis, establishing bondedness of $S(k)$, because this will be needed in what follows.

Soundararajan [11] remarks that "the unfortunate inequality" $S(k)<4 / k$ holds. This is not completely obvious, but in fact the situation is even worse, namely, $S(k)<4 /(k+$ $c \log k$ ). This was essentially proved (without an explicit calculation of $c$ ) in the mentioned unpublished note of Soundararajan [10]. Together with the mentioned example $P(x)=$ $x^{k+\ell}, \ell=c \sqrt{k}$, this shows that the value of $S(k)$ is between $4 /\left(k+c^{\prime} \log k\right)$ and $4 /(k+$ $\left.c^{\prime \prime} \sqrt{k}\right)$.

Proposition 1 (Soundararajan). The extremal problem (11) is bounded by $4 / k$. Moreover, we have $S(k)<\frac{4}{k+\log _{2} k-5}$ for all $k \geq 4$.

Proof. Let us fix, as in (4), the value $n:=k-1$. We are to show that whenever the denominator of (4) exists finitely, but is nonzero (i.e. when $P^{(k)}=Q^{\prime} \neq \mathbf{0}$ ), then also the numerator (with the condition that $Q(0)=0$, i.e. $P^{(k-1)}(0)=0$ ) exists finitely and, moreover, the ratio admits the stated bounds. 
Let us write now $q(x):=Q(1-x)$, assume that $Q$, hence also $q$, are absolutely continuous, and consider the resulting relations $q^{\prime}(x)=-Q^{\prime}(1-x), q(1)=Q(0)=0$. These imply by absolute continuity that $q(x)=q(x)-q(1)=-\int_{x}^{1} q^{\prime}(t) d t=\int_{x}^{1} Q^{\prime}(1-y) d y=$ $[-Q(1-y)]_{x}^{1}=Q(1-x)$-so we can as well start with the conditions that $p(x):=q^{\prime}(x)$ is measurable and finite a.e., admits the weighted bound $L:=\int_{0}^{1} x^{n} q^{\prime 2}(x) d x<\infty$ (coming from the requirement that the denominator is finite), and also that $q^{\prime}$ does not vanish a.e. (for the denominator being positive). First let us check that then defining $q$ from the given $p:=q^{\prime}$ as $q(x):=-\int_{x}^{1} q^{\prime}(t) d t$ works, results in an absolutely continuous function, and with this function the numerator stays finite, bounded in terms of $L$.

Indeed, $\int_{x}^{1}\left|q^{\prime}(t)\right| d t \leq \sqrt{\int_{x}^{1} t^{-n} d t \int_{x}^{1} t^{n}\left|q^{\prime}(t)\right|^{2} d t} \leq \sqrt{\frac{x^{1-n}}{n-1} \int_{0}^{1} t^{n}\left|q^{\prime}(t)\right|^{2} d t}=\sqrt{\frac{L}{n-1}} x^{\frac{1-n}{2}}$ (valid for all $n \geq 2$ ) gives not only that $q(x)$, as a Lebesgue integral, exists for all $x$, but also the estimate $|q(x)|^{2} \leq \frac{L}{n-1} x^{1-n}$ on $(0,1]$. It follows that $q(x)$ is absolutely continuous with derivative $p=q^{\prime}$ a.e. Moreover, $\int_{0}^{1} n x^{n-1} q^{2}(x) d x \leq \frac{n}{n-1} L$, too, hence the numerator in (44) is also finite and the quotient cannot exceed $\frac{k-1}{k-2} \leq 2(k \geq 3)$.

For $k=2$, i.e. $n=1$, there is only a little difference in the calculation, as then we obtain $|q(x)| \leq \sqrt{L|\log x|}$ and $\int_{0}^{1} n x^{n-1} q^{2}(x) d x=\int_{0}^{1} q^{2}(x) d x \leq L \int_{0}^{1}(-\log x) d x=L<2 L$, extending the above bound to all $k \geq 2$, too.

In the following we compute the stated sharper bound, too. For any $m \geq n-1$, $I(m):=\int_{0}^{1} x^{m} q^{2}(x) d x \leq \int_{0}^{1} x^{n-1} q^{2}(x) d x \leq 2 L<\infty$, as by condition we consider the class of functions satisfying $0<L<\infty$ (with $L:=\int_{0}^{1} x^{n} q^{\prime 2}(x) d x$ ).

Partial integration (using also $q(1)=Q(0)=0$ ) and Cauchy-Schwarz inequality yield

$$
I(m)=\frac{-1}{m+1} \int_{0}^{1} x^{m+1} 2 q^{\prime}(x) q(x) d x \leq \frac{2}{m+1} \sqrt{I(2 m+2-n) L} .
$$

So starting from $m:=m_{0}:=k-2$ and continuing by induction with $m_{j}:=k+2^{j}-3$ $(j=0,1, \ldots, N)$, we arrive at

$$
\frac{\int_{0}^{1} x^{k-2} q^{2}(x) d x}{\int_{0}^{1} x^{k-1} q^{\prime 2}(x) d x}=\frac{I\left(m_{0}\right)}{L} \leq \prod_{j=0}^{N}\left(\frac{2}{k+2^{j}-2}\right)^{2^{-j}} \cdot \frac{I\left(k+2^{N+1}-3\right)^{2^{-(N+1)}}}{L^{2^{-(N+1)}}} .
$$

Since $0 \leq I(\nu)$ is decreasing with $\nu, I\left(k+2^{N+1}-3\right)$ converges with $N$ so that we can pass to the limit $N \rightarrow \infty$, and then even take supremum with respect to $q$, obtaining

$S(k) \leq(k-1) \prod_{j=0}^{\infty}\left(\frac{2}{k+2^{j}-2}\right)^{2^{-j}}=\frac{4}{\prod_{j=1}^{\infty}\left(k+2^{j}-2\right)^{2^{-j}}}=\frac{4}{k-2} \prod_{j=1}^{\infty}\left(1+\frac{2^{j}}{k-2}\right)^{-2^{-j}}$.

Observe that for all $j \geq 1$ every single $k+2^{j}-2 \geq k$ in the denominator of the last but one expression, hence $S(k) \leq 4 / k$ follows immediately. We can even sharpen this estimate further. Let us denote the last product by $D:=D(k)$ and define $\ell:=\left[\log _{2}(k-2)\right]$. Then, 
by using $\log (1+x)>x-\frac{1}{2} x^{2}$ (for $x>0$ ) we infer

$$
\begin{aligned}
\log D(k)=-\sum_{j=1}^{\infty} \frac{\log \left(1+\frac{2^{j}}{k-2}\right)}{2^{j}} & <-\sum_{j=1}^{\ell} \frac{1}{2^{j}}\left(\frac{2^{j}}{k-2}-\frac{1}{2}\left(\frac{2^{j}}{k-2}\right)^{2}\right) \\
& =-\frac{\ell}{k-2}+\frac{2^{\ell}-1}{(k-2)^{2}}<-\frac{\ell-1}{k-2} .
\end{aligned}
$$

Therefore, as $e^{-x}<\frac{1}{1+x}($ for $x>0$ ), we obtain

$$
S(k)<\frac{4}{k-2} \cdot \exp \left(-\frac{\ell-1}{k-2}\right)<\frac{4}{(k-2)\left(1+\frac{\ell-1}{k-2}\right)}=\frac{4}{k+\ell-3} \leq \frac{4}{k+\log _{2} k-5},
$$

since $\ell \geq \log _{2}(k-2)-1 \geq \log _{2} k-2$ for all $k \geq 4$.

A further elementary observation is that for the Cauchy-Schwarz inequality to be precise, we should have $x^{m+1-n / 2} q=c x^{n / 2} q^{\prime}$ in all the above applications of the CauchySchwarz estimate (i.e., for all occurring values of $m$ ). This cannot hold for whatever choice of $q$ for all $m$ simultaneously. To have an about optimal estimate we may strive for having the Cauchy-Schwarz estimate sharp at the very first application, when $m=k-2$ and $n=k-1$, so $q=c q^{\prime}$ follows, and then $q(x)=e^{c x}$. But even that is not a valid choice in our problem: $q(1)=0$ prevents us taking $q$ an exponential function as it can never be zero. In any case, the estimate of $S(k)$ above cannot be sharp.

\section{Existence And SMoothness of MAXimizers in the EXtremal PRoblem}

3.1. Existence of maximizing functions in the extremal problem. In this paper the role of $k$ is fixed. Furthermore, it will be convenient for us to avoid repetitious use of $k-2$ and $k-1$, so throughout the rest of the paper except for the last section, Section 6. we will fix the notations for two further integer parameters. So we define

$$
m:=k-2, \quad n:=k-1 .
$$

As it is explained above, we can discuss the optimization problem in the function space

$$
\mathcal{Y}:=\left\{p(t) \in L_{\mathrm{loc}}^{1}(0,1]: \int_{0}^{1} p^{2}(t) t^{n} d t<\infty\right\}, \quad \text { where } q(x)=-\int_{x}^{1} p(t) d t .
$$

Multiplying the occurring functions by $t^{n / 2}$, we can even consider the space of functions $\varphi(t):=p(t) t^{n / 2}=q^{\prime}(t) t^{n / 2}$, which then will be square-integrable on $[0,1]$, so that $\varphi \in$ $L^{2}[0,1]$.

Next let us establish, how the functional to be maximized looks like over these spaces. On $\mathcal{X}$, on $\mathcal{Y}$ and finally on $L^{2}[0,1]$ we must consider the respective equivalent expressions

$$
\begin{aligned}
& \frac{-2 \int_{0}^{1} x^{k-1} q^{\prime}(x) q(x) d x}{\int_{0}^{1} x^{k-1} q^{\prime 2}(x) d x}=\frac{2 \int_{0}^{1} x^{n} p(x)\left(\int_{x}^{1} p(t) d t\right) d x}{\int_{0}^{1} p^{2}(x) x^{n} d x} \\
& \quad=\frac{2 \int_{0}^{1} \varphi(x) x^{n / 2}\left(\int_{x}^{1} \varphi(t) t^{-n / 2} d t\right) d x}{\int_{0}^{1} \varphi^{2}(x) d x}=\frac{2 \int_{0}^{1} \int_{0}^{1} \varphi(x) \varphi(t) \chi_{t>x}(x, t) x^{n / 2} t^{-n / 2} d t d x}{\int_{0}^{1} \varphi^{2}(x) d x}
\end{aligned}
$$

$$
=\frac{\int_{0}^{1} \int_{0}^{1} \varphi(x) \varphi(t) K(x, t) d t d x}{\int_{0}^{1} \varphi^{2}(x) d x} \quad \text { with } \quad K(x, t):=\left(\frac{\min (x, t)}{\max (x, t)}\right)^{n / 2}
$$


the last step being a technical one to bring the kernel $K$ to a symmetric form. So finally we find that

$$
S(k)=\sup _{L^{2}[0,1] \backslash\{0\}} \frac{\int_{0}^{1} \int_{0}^{1} \varphi(x) \varphi(t) K(x, t) d t d x}{\int_{0}^{1} \varphi^{2}(x) d x} \text { with } K(x, t):=\left(\frac{\min (x, t)}{\max (x, t)}\right)^{n / 2} .
$$

Clearly, on $L^{2}[0,1]$ the functional in (10) is defined everywhere except $\varphi=\mathbf{0}$ (the zero function), and is bounded by $4 / k$, as proved before. Moreover, there is a clear homogeneity property: The ratio for any $\varphi$ is equal to the ratio for any nonzero constant multiple $c \varphi$, hence the ratio is constant on all rays $\{c \varphi: c \in \mathbb{R}, c \neq 0\}$.

Therefore, the range of this quotient functional is clearly the same on the whole space $L^{2}[0,1] \backslash\{\mathbf{0}\}$ as on $B \backslash\{\mathbf{0}\}, B$ denoting the unit ball $B:=\left\{\varphi \in L^{2}[0,1]:\|\varphi\|_{2} \leq 1\right\}$ (where the 2-norm of a function in $L^{2}[0,1]$ is $\|\varphi\|_{2}:=\left(\int_{0}^{1} \varphi^{2}(x) d x\right)^{1 / 2}$, as usual). Furthermore, actually already on the unit sphere $S:=\left\{\varphi \in L^{2}[0,1]:\|\varphi\|_{2}=1\right\}$ the functional must take on all the values of its range. However, on the unit sphere the denominator is exactly one, so now we can modify the formulation and write

$$
S(k)=\sup _{S} \int_{0}^{1} \int_{0}^{1} \varphi(x) \varphi(t) K(x, t) d t d x=\sup _{B} \int_{0}^{1} \int_{0}^{1} \varphi(x) \varphi(t) K(x, t) d t d x .
$$

Moreover, it is clear that in this last formulation $S(k)$ is taken by a maximizer function $\varphi \in L^{2}[0,1]$ iff there is a maximum at some $\varphi \in S$ iff there is a maximum on $B$ (in which case again any maximum must belong to $S$ ). So any maximizer $\varphi$ in the original formulation is maximizer together with all the ray $\{c \varphi\}$ of its homothetic copies, and in the new formulation this maximizer occurs exactly with $c= \pm 1 /\|\varphi\|_{2}$, i.e., at the unit norm elements of the given ray.

This reformulation furnishes us the access to settle the existence question of some maximizer. In our formulation of the extremal problem all functions are real-valued, only for the next two propositions (spectral theory), and for the sake of being precise, we shall need complex-valued functions.

Proposition 2. Let

$$
K(x, y):=\left(\frac{\min (x, y)}{\max (x, y)}\right)^{n / 2}
$$

and define the Fredholm-type operator

$$
T: L^{2}([0,1] ; \mathbb{C}) \rightarrow L^{2}([0,1] ; \mathbb{C}) \quad(T \varphi)(x):=\int_{0}^{1} \varphi(y) K(x, y) d y .
$$

Then $T$ is a compact, positive, self-adjoint operator on the complex Hilbert space $L^{2}([0,1] ; \mathbb{C})$, maps real-valued functions into real-valued ones, and preserves positivity.

Proof. Since $K \in L^{\infty}([0,1] \times[0,1]), T$ is compact, see [9, $\left.\S 97\right]$. Since $0 \leq K \leq 1$ and $K$ is symmetric, the other two properties follow evidently.

Proposition 3. $S(k)$ is attained as a maximum by some maximizing function $\varphi \in L^{2}[0,1]$. Equivalently,

$$
S(k)=\frac{-2 \int_{0}^{1} x^{k-1} q^{\prime}(x) q(x) d x}{\int_{0}^{1} x^{k-1} q^{\prime 2}(x) d x}
$$

for some appropriate $q \in \mathcal{X}$ with $q(1)=0$ and $q \not \equiv \mathbf{0}$. 
Proof. Consider the operator $T$ as in (12).

$$
A(\varphi, \psi):=\int_{0}^{1} \int_{0}^{1} \varphi(y) \bar{\psi}(x) K(x, y) d x d y=\langle T \varphi, \psi\rangle,
$$

which is a sesquilinear form on $L^{2}([0,1] ; \mathbb{C})$. By [9, §93] we have

$$
\|T\|:=\sup _{\|\varphi\|_{2} \leq 1}\|T \varphi\|=\sup _{\|\varphi\|_{2} \leq 1}|\langle T \varphi, \varphi\rangle|=\sup _{\|\varphi\|_{2} \leq 1} A(\varphi, \varphi) .
$$

Since $T$ is compact, positive and self-adjoint, all of its eigenvalues are nonnegative, moreover, the eigenvalues can be ordered in a decreasing null-sequence $\left(\lambda_{j}\right), \lambda_{1}>\cdots>\lambda_{j}>$ $\ldots, \lambda_{j} \rightarrow 0(j \rightarrow \infty)$, and we also have

$$
\|T\|=\max \{\lambda: \lambda \text { is an eigenvalue of } T\}=: \lambda_{1} .
$$

Since $T$ leaves the subspace of real-valued functions invariant, for any eigenvalue $\lambda \in \mathbb{R}$ of $T$ there is a real-valued eigenfunction. Summing up, $\|T\|=\lambda_{1}$, and there exists some (nonzero) eigenfunction $\varphi \in L^{2}[0,1]$ satisfying $\|\varphi\|=1$ and $\lambda_{1}=\|T\|=A(\varphi, \varphi)$, yielding a maximizer for $A(\varphi, \varphi)$ as asserted.

Remark 4. The above proof yields also the following important information: $S(k)$ is the largest eigenvalue $\lambda_{1}$ of $T$, and any (normalized) eigenfunction $\varphi$ of $T$ belonging to $\lambda_{1}$ is a maximizer; moreover, the only maximizers are nonzero eigenfunctions of $T$ corresponding to $\lambda_{1}=\|T\|$.

Indeed, as $T$ is compact and self-adjoint, there is an orthonormal basis $\left(e_{j}\right)$ in $L^{2}[0,1]$ that consists of eigenfunctions of $T$. Let $\mathbf{0} \neq \varphi \in L^{2}[0,1]$ be not an eigenfunction to the eigenvalue $\lambda_{1}$. Then $\varphi=\sum_{j=1}^{\infty}\left\langle\varphi, e_{j}\right\rangle e_{j}$ and

$$
\langle T \varphi, \varphi\rangle=\sum_{j=1}^{\infty} \lambda_{j}\left|\left\langle\varphi, e_{j}\right\rangle\right|^{2}<\lambda_{1}\|\varphi\|_{2}
$$

by Parseval's identity, where for the strict inequality " $<$ " we have used that for some $j>1$ we have $\left|\left\langle\varphi, e_{j}\right\rangle\right|>0$, while $\lambda_{j}<\lambda_{1}$.

We also remark that since $K$ is strictly positive, so is the operator $T$, hence one knows from Perron-Frobenius theory (see [7, Sec. 4.2]) that the dominant eigenvalue $\lambda_{1}$ is simple with a corresponding strictly positive eigenfunction. This will be proved later also by directly determining all eigenfunctions of $T$.

Next we turn to smoothness properties of eigenfunctions of $T$.

3.2. Smoothness of maximizers and maximizers in $C[0,1]$. The above formulation also provides us a direct access to further smoothness statements.

Lemma 5. The Fredholm-type operator $T$ defined in (12) maps $L^{2}[0,1]$ to the subspace $C_{0}(0,1]$ of continuous functions with value 0 at 02 .

Proof. Since $L^{2}[0,1] \subset L^{1}[0,1]$, and $0 \leq K(x, y) \leq 1$, the expression $(T \varphi)(x)=\int_{0}^{1} \varphi(y) K(x, y) d y$ is an integral with a uniform majorant $|\varphi(y)| \in L^{1}[0,1]$ of the integrands. Hence by the Lebesgue Dominated Convergence Theorem, it suffices to take the pointwise limit under the integral sign. When $x \rightarrow x_{0}$, this gives for all $y>0 \lim _{x \rightarrow x_{0}} K(x, y)=K\left(x_{0}, y\right)$, while for $y=0$ we have $K(x, 0)=K\left(x_{0}, 0\right)=0$ identically. (Essentially, we have used only separate continuity of $K$ on $[0,1] \times[0,1]$.) Thus $\lim _{x \rightarrow x_{0}}(T \varphi)(x)=\int_{0}^{1} \varphi(y) \lim _{x \rightarrow x_{0}} K(x, y) d y=$

\footnotetext{
${ }^{2}$ Note that we identify functions defined on $(0,1]$ only but having limit 0 towards the boundary point 0 with functions continuously extended to 0 by defining their value at 0 as 0 .
} 
$\int_{0}^{1} \varphi(y) K\left(x_{0}, y\right) d y=(T \varphi)\left(x_{0}\right)$, i.e., $T \varphi \in C[0,1]$. By definition, $K(0, y)=0$ for all $y \in[0,1]$, hence for every $\varphi \in L^{2}[0,1]$ we have $(T \varphi)(0)=0$.

Corollary 6. All eigenfunctions $\varphi$ of the Fredholm-type operator $T$ defined in (12) are continuous and fulfill $\varphi(0)=0$.

Equivalently, in the function space $\mathcal{X}$ defined in (7) all the functions $q(x)=-\int_{x}^{1} q^{\prime}(t) d t=$ $-\int_{x}^{1} t^{-n / 2} \varphi(t) d t$ corresponding to eigenfunctions $\varphi$ of $T$ satisfy $\varphi(x)=x^{n / 2} q^{\prime}(x) \in C_{0}(0,1]$ and thus $x^{n / 2-1} q(x) \in C_{0}(0,1]$.

Proof. All eigenfunctions lie in the range of the operator $T$, hence belong to $C_{0}(0,1]$ in view of Lemma 5 ,

Recall that the correspondence between $L^{2}[0,1]$ and our spaces $\mathcal{Y}$ and $\mathcal{X}$ was given by $\varphi(x)=x^{n / 2} p(x)=x^{n / 2} q^{\prime}(x)$. Thus for $\varphi \in L^{2}[0,1]$, an eigenfunction of $T$, we obtain for the corresponding $q$ that $x^{n / 2} q^{\prime}(x) \in C_{0}(0,1]$, whence also $q^{\prime} \in C(0,1]$ follows. Moreover, $\lim _{x \rightarrow 0+} x^{n / 2} q^{\prime}(x)=\lim _{x \rightarrow 0+} \varphi(x)=\varphi(0)=0$, providing a continuous extension of $x^{n / 2} q^{\prime}(x)$ even to 0 . Now writing $q(x)=-\int_{x}^{1} q^{\prime}(t) d t$ yields $q \in C(0,1]$. While for $x \rightarrow 0+$ we obtain that ${ }^{3}$

$$
\lim _{x \rightarrow 0+} x^{n / 2-1} q(x)=\lim _{x \rightarrow 0+} x^{n / 2-1}\left(-\int_{x}^{1} o(1) t^{-n / 2} d t\right)=\lim _{x \rightarrow 0+} o(1) \frac{2}{n-2}=0,
$$

hence $x^{n / 2-1} q(x) \in C_{0}(0,1]$.

Although $K$ is not everywhere continuous on $[0,1] \times[0,1]$, the operator $T$ can still be approximated by compact operators given by continuous kernels.

Proposition 7 . The operator $T$ restricted to $C[0,1]$ is a compact $C[0,1] \rightarrow C[0,1]$ operator with exactly the same eigenvalues and eigenfunctions as on $L^{2}[0,1]$.

Proof. Let $f_{j}:[0,1] \times[0,1] \rightarrow[0,1]$ be continuous with $f_{j}(x, y)=0$ if $x, y \leq \frac{1}{2 j}$ and $f_{j}(x, y)=1$ if $\max (x, y) \geq \frac{1}{j}$. Then $f_{j} K$ is continuous, hence the integral operator $T_{j}$ with kernel $f_{j} K$ is compact, see, e.g., [9, §90]. It is easy to see that $T_{j} \rightarrow T$ in the operator norm (over $C[0,1]$ ), hence $T$ itself is compact, see [9, $\S 76]$.

By Proposition 6 all eigenfunctions of $T$ on $L^{2}[0,1]$ belong also to $C_{0}(0,1]$, hence remain eigenfunctions when $T$ is considered as $C[0,1] \rightarrow C[0,1]$. The converse is obvious: Every continuous eigenfunction is of course also an eigenfunction from $L^{2}[0,1]$. In particular, the set of eigenvalues are also exactly the same when considered in these two spaces.

Remark 8. One can show that the norm of $T$ as an operator on $C[0,1]$ is

$$
\|T\|_{C[0,1]}=\left(\frac{4}{n+2}\right)^{\frac{n}{n-2}}=\left(\frac{4}{k+1}\right)^{\frac{k-1}{k-3}} .
$$

In fact, since $T$ is positivity preserving, we have $\|T\|_{C[0,1]}=\|T \mathbf{1}\|_{\infty}$, where $\mathbf{1}$ is the constant 1 function. Easy calculation shows that $(T \mathbf{1})(x)=\frac{4 n}{n^{2}-4} x-\frac{2}{n-2} x^{n / 2}$ and that this function has maximum at $x=\left(\frac{2}{n+2}\right)^{2 /(n-2)}$. Since we already know $\|T\|_{L^{2}[0,1]}=\lambda_{1}$, and in

\footnotetext{
${ }^{3}$ Here $o(1)$ means that for any $\varepsilon>0$ we have some $\delta$ such that for $0 \leq t \leq \delta,\left|q^{\prime}(t)\right|<\varepsilon t^{-n / 2}$. Therefore, we have for any $0<x<\delta$ the estimate $|q(x)-q(\delta)| \leq \int_{x}^{\delta} \varepsilon t^{-n / 2} d t<\frac{2 \varepsilon}{n-2} x^{1-n / 2}$, while $x^{n / 2-1} q(\delta) \rightarrow 0$ as $x \rightarrow 0+$. Therefore, $\left|x^{n / 2-1} q(x)\right| \leq \frac{2 \varepsilon}{n-2}+o(1)$ and finally $x^{n / 2-1} q(x) \rightarrow 0$ with $x \rightarrow 0+$.
} 
general $\lambda_{1} \leq\|T\|_{C[0,1]}$, we obtain that the maximum of $A(\varphi, \varphi)$ with $\|\varphi\|_{2}=1$, i.e., $S(k)$ is smaller than the constant in (14) above.

3.3. Differentiability of maximizers. We now push further the smoothness statements from the last subsection. We need some preparations, and define the following auxiliary functions

$$
\kappa(x, y):= \begin{cases}\frac{\min (x, y)}{\max (x, y)} & \text { if } \quad(0,0) \neq(x, y) \in[0,1]^{2} \\ 0 & \text { if } \quad(x, y)=(0,0)\end{cases}
$$

and

$$
\omega(a, b):= \begin{cases}\frac{\sum_{j=0}^{n-1} a^{j / 2} b^{(n-1-j) / 2}}{\sqrt{b}+\sqrt{a}} & \text { if } \quad(0,0) \neq(a, b) \in[0,1]^{2}, \\ 0 & \text { if } \quad(a, b)=(0,0) .\end{cases}
$$

With these notations we have for every $0<x, y \leq 1$ the formula

$$
\frac{n}{2} K(x, y)=\omega(\kappa(x, y), \kappa(x, y)) \kappa(x, y),
$$

which also holds for $x$ or $y$ being 0 , with both sides vanishing.

Now note that $0 \leq \omega(a, b) \leq n \max (a, b)^{n / 2-1} \leq n$. Furthermore, observe that for $0 \leq a, b \leq 1$ (even if both are zero) we have $b^{n / 2}-a^{n / 2}=(\sqrt{b}-\sqrt{a}) \sum_{j=0}^{n-1} a^{j / 2} b^{(n-1-j) / 2}=$ $(b-a) \omega(a, b)$. Hence for any $y, x, x^{\prime} \in[0,1]$ we can write

$$
K\left(x^{\prime}, y\right)-K(x, y)=\omega\left(\kappa\left(x^{\prime}, y\right), \kappa(x, y)\right) \cdot\left(\kappa\left(x^{\prime}, y\right)-\kappa(x, y)\right) .
$$

Fix $x>0$. Denoting $\Delta:=x^{\prime}-x>0$, we also have

$$
\kappa\left(x^{\prime}, y\right)-\kappa(x, y)= \begin{cases}\frac{y}{x^{\prime}}-\frac{y}{x}=-\frac{y \Delta}{x x^{\prime}} & \text { if } y<x<x^{\prime}, \\ \frac{y}{x^{\prime}}-\frac{x}{y}=\frac{y^{2}-x x^{\prime}}{y x^{\prime}} & \text { if } x \leq y \leq x^{\prime}, \\ \frac{x^{\prime}}{y}-\frac{x}{y}=\frac{\Delta}{y} & \text { if } x<x^{\prime}<y,\end{cases}
$$

whence

$$
\left|\kappa\left(x^{\prime}, y\right)-\kappa(x, y)\right|= \begin{cases}\frac{y}{x}-\frac{y}{x^{\prime}}=\frac{y \Delta}{x x^{\prime}} \leq \frac{\Delta}{x^{\prime}} \leq \frac{\Delta}{x} & \text { if } y<x<x^{\prime} \\ \left|\frac{x}{y}-\frac{y}{x^{\prime}}\right|=\frac{\left|x x^{\prime}-y^{2}\right|}{y x^{\prime}} \leq \frac{\Delta}{y} \leq \frac{\Delta}{x} & \text { if } x \leq y \leq x^{\prime} \\ \frac{x^{\prime}}{y}-\frac{x}{y}=\frac{\Delta}{y} \leq \frac{\Delta}{x^{\prime}} \leq \frac{\Delta}{x} & \text { if } x<x^{\prime}<y .\end{cases}
$$

Lemma 9. The operator $T$ maps $L^{2}[0,1]$ to the space $C^{1}(0,1]$. Furthermore, for $\varphi \in$ $L^{2}[0,1]$ and $x \in(0,1]$

$$
(T \varphi)^{\prime}(x)=\int_{0}^{1} \varphi(y) \frac{\partial}{\partial x} K(x, y) d y=-\frac{n}{2 x}(T \varphi)(x)+n x^{n / 2-1} \int_{x}^{1} \frac{\varphi(y)}{y^{n / 2}} d y .
$$

Proof. The two expressions given for $(T \varphi)^{\prime}(x)$ in (21) are easily seen to be equal, so the proof hinges upon showing that $(T \varphi)^{\prime}(x)$ equals any one of them.

For any $0 \leq x<x^{\prime} \leq 1$ using (18) we can write

$$
\frac{(T \varphi)\left(x^{\prime}\right)-(T \varphi)(x)}{x^{\prime}-x}=\int_{0}^{1} \varphi(y) \omega\left(\kappa\left(x^{\prime}, y\right), \kappa(x, y)\right) \frac{\kappa\left(x^{\prime}, y\right)-\kappa(x, y)}{x^{\prime}-x} d y .
$$

We fix $x_{0}>0$ and take either $x=x_{0}$ and $x^{\prime} \rightarrow x_{0}+$, or $x^{\prime}=x_{0}$ and $x \rightarrow x_{0}-$. In any case, by (20) and $0 \leq \omega \leq n$ we have the Lebesgue integrable majorant $n|\varphi(y)| / x_{0}$ of the integrand, thus limit and integral can be interchanged. 
For example in the case $x^{\prime} \rightarrow x_{0}+$ taking into account (17) we are led to

$$
\begin{aligned}
& \lim _{x^{\prime} \rightarrow x_{0}} \frac{(T \varphi)\left(x^{\prime}\right)-(T \varphi)\left(x_{0}\right)}{x^{\prime}-x_{0}}=\left.\int_{0}^{1} \varphi(y) \omega\left(\kappa\left(x_{0}, y\right), \kappa\left(x_{0}, y\right)\right) \frac{\partial}{\partial x} \kappa(x, y)\right|_{x=x_{0}} d y \\
& \quad=\int_{0}^{1} \varphi(y) \omega\left(\kappa\left(x_{0}, y\right), \kappa\left(x_{0}, y\right)\right) \operatorname{sgn}\left(y-x_{0}\right) \frac{\kappa\left(x_{0}, y\right)}{x_{0}} d y \\
& \quad=\frac{n}{2 x_{0}} \int_{0}^{1} \varphi(y) K\left(x_{0}, y\right) \operatorname{sgn}\left(y-x_{0}\right) d y=\frac{n}{2 x_{0}}\left(\int_{x_{0}}^{1} \varphi(y) K\left(x_{0}, y\right) d y-\int_{0}^{x_{0}} \varphi(y) K\left(x_{0}, y\right) d y\right)
\end{aligned}
$$

$$
=\frac{n}{x_{0}}\left(\int_{x_{0}}^{1} \varphi(y) K\left(x_{0}, y\right) d y-\frac{1}{2}(T \varphi)\left(x_{0}\right)\right),
$$

where we have used $\frac{\partial}{\partial x} \kappa(x, y)=\frac{\partial}{\partial x}(x / y)=1 / y$ for $y>x$ and $\frac{\partial}{\partial x} \kappa(x, y)=\frac{\partial}{\partial x}(y / x)=$ $-y / x^{2}$ for $x<y$. When substituting the definition of $K$ in the above, we obtain all the asserted formulas. Note that in case $y=x_{0}$, one sided derivatives of $\kappa\left(x_{0}, y\right)$ still exist (and are equal to the limits from the respective side) but the existence and value of the limit at one exceptional point does not interfere the value of the integral, therefore we have just put 0 for the value of $\left.\frac{\partial}{\partial x} \kappa\left(x, x_{0}\right)\right|_{x=x_{0}}$ here.

When $x \rightarrow x_{0}-=x^{\prime}-$, the calculation is entirely the same.

The integrals on the right hand side of (21) are of course integrals of integrable functions, and as such, are continuous in function of the limits of integration. Therefore, continuity of $(T \varphi)^{\prime}$ on $(0,1]$ also follows.

Remark 10. When $x_{0}=0$, only the right hand side derivative can be considered and thus we take $x_{0}=x=0$ and $x^{\prime} \rightarrow 0+$. Also, $(T \varphi)(0)=0$ and $K(0, y)=0$, hence the consideration of the differential reduces to

$$
\lim _{x^{\prime} \rightarrow 0+} \frac{(T \varphi)\left(x^{\prime}\right)}{x^{\prime}}=\lim _{x^{\prime} \rightarrow 0+} \frac{1}{x^{\prime}} \int_{0}^{1} \varphi(y) K\left(x^{\prime}, y\right) d y=\lim _{x^{\prime} \rightarrow 0+} \int_{0}^{1} \varphi(y) \frac{\kappa^{n / 2}\left(x^{\prime}, y\right)}{x^{\prime}} d y
$$

which, however, cannot be handled for general $\varphi \in L^{2}[0,1]$ or not even for $\varphi \in C_{0}(0,1]$, and can be well estimated only if we use something more on $\varphi$. See Corollary $12 \mathrm{~g}$ below.

Proposition 11. The operator $T$ maps $L^{2}[0,1]$ to the space of absolutely continuous functions with bounded total variation. Moreover, for the total variation of $T \varphi$ we have $V(T \varphi,[0,1]) \leq 2\|\varphi\|_{1}$.

Proof. We already know that $T \varphi \in C^{1}(0,1]$, so the total variation, whether finite or infinite, can be computed as $V(T \varphi,[0,1])=\int_{0}^{1}\left|(T \varphi)^{\prime}\right|$. Now the first formula from (21) furnishes

$$
\begin{aligned}
V(T \varphi,[0,1]) & =\int_{0}^{1}\left|(T \varphi)^{\prime}(x)\right| d x=\int_{0}^{1}\left|\int_{0}^{1} \varphi(y) \frac{\partial}{\partial x} K(x, y) d y\right| d x \\
& \leq \int_{0}^{1} \int_{0}^{1}|\varphi(y)|\left|\frac{\partial}{\partial x} K(x, y)\right| d y d x=\int_{0}^{1}|\varphi(y)|\left(\int_{0}^{1}\left|\frac{\partial}{\partial x} K(x, y)\right| d x\right) d y \leq 2\|\varphi\|_{1},
\end{aligned}
$$

as for all $0<y<1$ fixed we have $\int_{0}^{1}\left|\frac{\partial}{\partial x} K(x, y)\right| d x=V(K(\cdot, y),[0,1])=2-y^{n / 2}<2$.

Corollary 12. If $\psi$ lies in the range of $T$, then $T \psi \in C^{1}[0,1]$, and $(T \psi)^{\prime}(0)=0$. In particular if $\varphi$ is an eigenfunction of $T$, then $\varphi \in C^{1}[0,1]$, and $\varphi^{\prime}(0)=0$. 
Proof. We have to calculate the limit in (24) for $\psi:=T \varphi$ in place of $\varphi$. Recall from the above that then $\psi=T \varphi \in C_{0}(0,1]$, in particular $\psi(0)=(T \varphi)(0)=0$, and $\psi \in C^{1}(0,1]$, $V(\psi,[0,1]) \leq 2\|\varphi\|_{1}$. The second mean value theorem and integration by parts yield with some appropriate $z:=z_{x^{\prime}} \in\left(0, x^{\prime}\right)$

$$
\begin{aligned}
& \frac{1}{x^{\prime}} \int_{0}^{1} \psi(y) K\left(x^{\prime}, y\right) d y=\frac{1}{x^{\prime}}\left\{\int_{0}^{x^{\prime}} \psi(y) K\left(x^{\prime}, y\right) d y+\int_{x^{\prime}}^{1} \psi(y) \frac{x^{\prime n / 2}}{y^{n / 2}} d y\right\} \\
&=\psi\left(z_{x^{\prime}}\right) K\left(x^{\prime}, z_{x^{\prime}}\right)+\left\{\left[\psi(y) \frac{-x^{\prime n / 2-1}}{n / 2-1} y^{1-n / 2}\right]_{x^{\prime}}^{1}+\int_{x^{\prime}}^{1} \psi^{\prime}(y) \frac{x^{\prime n / 2-1}}{n / 2-1} y^{1-n / 2} d y\right\},
\end{aligned}
$$

so the first term tends to $\psi(0)=0$ when $x^{\prime} \rightarrow 0+$. The term in the square bracket contributes $x^{\prime n / 2-1} \frac{-\psi(1)}{n / 2-1}+\frac{\psi\left(x^{\prime}\right)}{n / 2-1}$, and as $x^{\prime} \rightarrow 0+$ and $\psi\left(x^{\prime}\right) \rightarrow \psi(0)=0$, both terms converge to 0. Finally, for the integral Proposition 11 gives that $\psi^{\prime} \in L^{1}[0,1]$, while the product of the further factors stays bounded uniformly for all $x^{\prime}, y \in[0,1]$, as the integral runs only through values $y \geq x^{\prime}$. That is, we can again use the Lebesgue Dominated Convergence Theorem and calculate the limit by moving it under the integral sign. Furthermore, the pointwise limit of the expression is zero for all fixed $y$, whence the assertion follows.

\section{Solving the MaXimization PROBlem}

4.1. Setting up a differential equation for potential extremal functions. By the previous section we know that our maximization problem has a solution, and we also saw that maximizers are sufficiently smooth. We can now set up a differential equation to find maximizers, or which is essentially equivalent, to find the eigenfunctions of $T$.

Proposition 13. Let $\varphi \in L^{2}[0,1]$ be an eigenfunction of $T$ corresponding to the eigenvalue $\lambda>0$. Then $\varphi$ is continuous on $[0,1]$, infinitely often differentiable on $(0,1]$. The function $q(x)=-\int_{x}^{1} \varphi(y) y^{-n / 2} d y$ satisfies $q(1)=0$ and the differential equation

$$
q^{\prime \prime}(x)+\frac{n}{x} q^{\prime}(x)+\frac{b}{x} q(x)=0 \quad(x \in(0,1]), \quad \text { where } \quad b:=\frac{n}{\lambda}>0 .
$$

Conversely, let $\lambda>0$ and suppose that $q$ is a nonzero, $C^{2}(0,1]$ solution of the differential equation above with $q(1)=0$. If $\varphi(x)=x^{n / 2} q^{\prime}(x)$ extends continuously to 0 with $\lim _{x \rightarrow 0+} x^{n / 2} q^{\prime}(x)=0$, then $\varphi$ is an eigenfunction of $T$ corresponding to the eigenvalue $\lambda>0$.

Proof. If $\varphi \in L^{2}[0,1]$ is an eigenfunction of $T$ for the eigenvalue $\lambda>0$, then it belongs to the range of $T$, hence is continuous and continuously differentiable on $(0,1]$ by Lemma 9 . Substituting $T \varphi=\lambda \varphi$ in (21) we obtain

$$
\lambda \varphi^{\prime}(x)=-\frac{n}{2 x} \lambda \varphi(x)+n x^{n / 2-1} \int_{x}^{1} \frac{\varphi(y)}{y^{n / 2}} d y .
$$

As the right-hand side is differentiable, we can differentiate also the left-hand side showing $\varphi \in C^{2}(0,1]$. We substitute $x^{-n / 2} \varphi(x)=q^{\prime}(x)$ and $\varphi(x)=x^{n / 2} q^{\prime}(x)$ and obtain

$$
\frac{d}{d x}\left(\lambda x^{n / 2} q^{\prime}(x)\right)=-\frac{n}{2 x} \lambda x^{n / 2} q^{\prime}(x)+n x^{n / 2-1} \int_{x}^{1} q^{\prime}(y) d y,
$$

and hence

$$
\frac{d}{d x}\left(\lambda x^{n / 2} q^{\prime}(x)\right)=-\lambda \frac{n}{2} x^{n / 2-1} q^{\prime}(x)-n x^{n / 2-1} q(x) .
$$


Differentiation yields

$$
\lambda x^{n / 2} q^{\prime \prime}(x)+\lambda \frac{n}{2} x^{n / 2-1} q^{\prime}(x)=-\frac{\lambda n}{2 x} x^{n / 2} q^{\prime}(x)-n x^{n / 2-1} q(x),
$$

and then by rearranging we obtain

$$
\lambda x^{n / 2} q^{\prime \prime}(x)+\lambda \frac{n}{x} x^{n / 2} q^{\prime}(x)+n x^{n / 2-1} q(x)=0 .
$$

Division by $\lambda x^{n / 2}$ thus leads to the asserted differential equation (25).

To see the converse we set $\psi=T \varphi$. Note that then $\psi \in C_{0}(0,1]$ according to Lemma 5 , Then $\varphi(x)=x^{n / 2} q^{\prime}(x)$ entails

$$
q^{\prime \prime}(x)=-\frac{n}{2} x^{-n / 2-1} \varphi(x)+x^{-n / 2} \varphi^{\prime}(x),
$$

so that using the assumption that $q$ solves (25) we obtain

$$
-\frac{n}{2} x^{-n / 2-1} \varphi(x)+x^{-n / 2} \varphi^{\prime}(x)+\frac{n}{x} x^{-n / 2} \varphi(x)+\frac{n / \lambda}{x}\left(-\int_{x}^{1} y^{-n / 2} \varphi(y) d y\right)=0,
$$

and thus also

$$
\frac{n}{2} \lambda x^{-n / 2-1} \varphi(x)+\lambda x^{-n / 2} \varphi^{\prime}(x)-\frac{n}{x} \int_{x}^{1} y^{-n / 2} \varphi(y) d y=0 .
$$

By (21) with $\psi=T \varphi$ we also have

$$
x^{-n / 2} \psi^{\prime}(x)=-\frac{n}{2} x^{-n / 2-1} \psi(x)+\frac{n}{x} \int_{x}^{1} \frac{\varphi(y)}{y^{n / 2}} d y,
$$

SO

$$
\frac{n}{2} \lambda x^{-n / 2-1} \varphi(x)+\lambda x^{-n / 2} \varphi^{\prime}(x)-x^{-n / 2} \psi^{\prime}(x)-\frac{n}{2} x^{-n / 2-1} \psi(x)=0 .
$$

If we multiply by $x^{n}$, we obtain for all $x>0$

$0=\frac{n}{2} \lambda x^{n / 2-1} \varphi(x)+\lambda x^{n / 2} \varphi^{\prime}(x)-x^{n / 2} \psi^{\prime}(x)-\frac{n}{2} x^{n / 2-1} \psi(x)=\frac{d}{d x}\left(x^{n / 2} \lambda \varphi(x)-x^{n / 2} \psi(x)\right)$.

Since $\varphi, \psi \in C[0,1], x^{n / 2}(\lambda \varphi(x)-\psi(x))$ must vanish at 0 , whence $\lambda \varphi=\psi$ follows.

Thus the solution of the maximization problem is reduced to solving the homogeneous second order ordinary differential equation (25), and to finding the feasible values of $\lambda$. Next we solve this equation and analyze some properties of the solutions.

4.2. Bessel functions and Bessel's differential equation. Recall Bessel's differential equation

$$
y^{\prime \prime}(x)+\frac{1}{x} y^{\prime}(x)+\left(1-\frac{\nu^{2}}{x^{2}}\right) y(x)=0,
$$

for some fixed parameter $\nu \in \mathbb{R}$. The Bessel function $J_{\nu}$ (of the first kind) is a solution of this equation, [2, (6.71), p. 115]. Notice that $J_{-\nu}$ is also a solution, but for $\nu$ integer $J_{\nu}$ and $J_{-\nu}$ are linearly dependent, in fact $J_{-\nu}=(-1)^{\nu} J_{\nu}$. So for $\nu \in \mathbb{N}$ another, linearly independent solution is needed, which is provided by the Bessel functions $Y_{\nu}$ (of the second kind), obtained as

$$
Y_{\nu}(x):=J_{\nu}(x) \int \frac{d x}{x J_{\nu}^{2}(x)},
$$

where specifying the limits of integration is equivalent to fix some primitive of the integrand, and the integration limit cannot be at 0 where $Y_{\nu}(x)$ is divergent in the order $x^{-\nu}$, 
see [2, (6.73), (6.74)]. Then for $\nu \in \mathbb{N}$ the general solution of equation (26) is a linear combination $c_{1} J_{\nu}+c_{2} Y_{\nu}$, see [2, $\left.\S 102\right]$.

4.3. Solution of the differential equation. Bowman computes, see [2, (6.80), p. 117], what happens if we consider the transformed, substituted functions $u(x):=x^{\alpha} y\left(\beta x^{\gamma}\right)$, where $y$ satisfies the Bessel equation (26), and establishes that then the new functions $u(x)$ will be the general solutions of the transformed equation

$$
u^{\prime \prime}(x)-\frac{2 \alpha-1}{x} u^{\prime}(x)+\left(\beta^{2} \gamma^{2} x^{2 \gamma-2}+\frac{\alpha^{2}-\nu^{2} \gamma^{2}}{x^{2}}\right) u(x)=0 .
$$

If we choose here the parameters $\nu:=m, \alpha:=-m / 2=1-k / 2, \beta:=2 \sqrt{n / \lambda}$ and $\gamma:=1 / 2$ (where $n:=k-1=m+1$ and $m:=k-2$ as fixed above in (8)), then the equation (27) becomes exactly (25). Thus we obtain that for any fixed values of $m:=k-2$ and $\lambda>0$, there is a one-to-one correspondence between the solutions $q$ of (25) and $y$ of (26) given by $q(x):=x^{\alpha} y\left(\beta x^{\gamma}\right)=x^{1-k / 2} y(2 \sqrt{n / \lambda} \sqrt{x})$.

Corollary 14. Every solution $q$ of (25) is a linear combination of transformed Bessel functions from the above, i.e.,

$$
q(x)=c_{1} x^{-m / 2} J_{m}(2 \sqrt{n / \lambda} \sqrt{x})+c_{2} x^{-m / 2} Y_{m}(2 \sqrt{n / \lambda} \sqrt{x}), \quad(m=n-1=k-2) .
$$

4.4. Some analysis of the occurring Bessel type functions. Before proceeding, let us note one important thing. Not all solutions of the differential equation are relevant for us, because the resulting $q^{\prime}$ must have finite weighted square integral, i.e., $\varphi(x)=x^{n / 2} q^{\prime}(x)$ has to belong to $L^{2}[0,1]$ (and even be continuous on $[0,1]$, according to Proposition [13).

According to the second formula of [1, 9.1.30] (with the choice $k=1$ and $\nu=m$ there) we have

$$
\begin{aligned}
& \left(\frac{1}{x} \frac{d}{d x}\right)\left(x^{-m} c_{1} J_{m}(x)+c_{2} Y_{m}(x)\right) \\
& \quad=-x^{-m-1}\left(c_{1} J_{m+1}(x)+c_{2} Y_{m+1}(x)\right)=-x^{-n}\left(c_{1} J_{n}(x)+c_{2} Y_{n}(x)\right),
\end{aligned}
$$

therefore we obtain for a solution $q$ of (25) that

$$
\begin{aligned}
q^{\prime}(x) & =\frac{d}{d x}\left(c_{1} x^{-m / 2} J_{m}(2 \sqrt{n / \lambda} \sqrt{x})+c_{2} x^{-m / 2} Y_{m}(2 \sqrt{n / \lambda} \sqrt{x})\right) \\
& =-\frac{1}{2 \sqrt{n / \lambda}} x^{1 / 2} x^{-n / 2}\left(c_{1} J_{n}(2 \sqrt{n / \lambda} \sqrt{x})+c_{2} Y_{n}(2 \sqrt{n / \lambda} \sqrt{x})\right) .
\end{aligned}
$$

So that

$$
\varphi(x)=x^{n / 2} q^{\prime}(x)=-\frac{\sqrt{x}}{2 \sqrt{n / \lambda}}\left(c_{1} J_{n}(2 \sqrt{n / \lambda} \sqrt{x})+c_{2} Y_{n}(2 \sqrt{n / \lambda} \sqrt{x})\right) .
$$

To see when such a $\varphi$ may actually be an eigenfunction of $T$, we first find out when it belongs to $C_{0}(0,1]$. First, see [2, (1.2)]

$$
J_{\nu}(x)=x^{\nu} \sum_{j=0}^{\infty} \frac{(-1)^{j}}{2^{\nu+2 j}} \frac{x^{2 j}}{j !(\nu+j) !}, \quad \text { whence also } \quad J_{\nu}(x) \sim \frac{1}{2^{\nu} \nu !} x^{\nu}(x \rightarrow 0+),
$$

so in particular $J_{n}$ is continuous on $[0, \infty)$, and for any $c_{1} \in \mathbb{R}$ the part $\frac{\sqrt{x}}{2 \sqrt{n / \lambda}} c_{1} J_{n}(2 \sqrt{n / \lambda} \sqrt{x})$ belongs to $C_{0}(0,1] \subset L^{2}[0,1]$. Second, $Y_{n}(x) \asymp x^{-n}(x \rightarrow 0+)$ [2, p. 116], entailing that for $c_{2} \neq 0 \frac{\sqrt{x}}{2 \sqrt{n / \lambda}} c_{2} Y_{n}(a \sqrt{x}) \asymp x^{-(n-1) / 2}$, and this function is not even bounded near 0 , if 
$n>1$ (i.e., when $k=n-1>2$ ). If $n=1$, i.e., $k=2$, this function is not vanishing at 0 , a condition that is necessary for an eigenfunction of $T$ by Lemma 5. So from Corollary 6 we obtain that $\varphi$ in (28) belongs to $C_{0}(0,1]$ (and thus may be a candidate for being an eigenfunction of $T$ ) if and only if $c_{2}=0$.

As a consequence of this and of Proposition 13 we obtain the following.

Corollary 15. Consider the operator $T$ from (12), and let $\lambda>0$. Then $\lambda>0$ is an eigenvalue of $T$ if and only if

$$
J_{m}(2 \sqrt{n / \lambda})=0
$$

In this case

$$
\varphi(x)=x^{n / 2} q^{\prime}(x)=c_{1} x^{1 / 2} J_{n}(2 \sqrt{n / \lambda} \sqrt{x}), \quad c_{1} \neq 0
$$

are the only eigenfunctions corresponding to $\lambda$.

For given $m$ let us denote the roots of $J_{m}$ by $\alpha_{m, r}(r \in \mathbb{N})$ ordered increasingly. At this stage it is in order to recall the following about the zeros of Bessel functions. We have $\alpha_{m, r} \rightarrow \infty(r \rightarrow \infty)$, and for rather large values the roots $\alpha_{m, r}$ of $J_{m}$ are very well distributed, as essentially there falls one root in each interval of length $\pi$. However, for fixed $m$ the increasing sequence of zeros $\left(\alpha_{m, r}\right)$ starts only with $\alpha_{m, 1} \sim m+\mathrm{cm}^{1 / 3}$, with $c=1.8557571 \ldots$, see [1, 9.5.14, p. 341]. Let us introduce the notation

$$
\lambda_{m, r}:=\lambda_{r}:=4(m+1) / \alpha_{m, r}^{2}
$$

and

$$
q_{r}(x):=q_{m, r}(x):=x^{-m / 2} J_{m}\left(2 \sqrt{(m+1) / \lambda_{m, r}} \cdot \sqrt{x}\right)=x^{-m / 2} J_{m}\left(\alpha_{m, r} \sqrt{x}\right) .
$$

By putting everything together we obtain the following result.

Theorem 16. For the extremal problem (1) we have

$$
S(k)=\lambda_{1}=\frac{4(k-1)}{\alpha_{k-2,1}^{2}}=\frac{4}{k+2 c k^{1 / 3}+O(1)}
$$

with $\alpha_{k-2,1}$ the first root of the order $k-2$ Bessel function $J_{k-2}(x)$, and the constant $c=1.8557571 \ldots$

The only extremal functions for the formulation (5) are nonzero constant multiples of

$$
q_{1}(x)=x^{-(k-2) / 2} J_{k-2}\left(\alpha_{k-2,1} \sqrt{x}\right) .
$$

Proof. Uniqueness follows from Remark 4 and Corollary 15. For $q_{1}$ being a maximizer for (5) it remains only to show that

$$
\int_{0}^{1} q_{1}(y) y^{n} d y \neq 0 .
$$

But this follows since $q_{1}$ is (strictly) positive all over $(0,1), \alpha_{k-2,1}$ being the very first zero of the Bessel function $J_{k-2}$. 


\section{POWER SERIES}

The above settles the issue of the best weight $P$-and also the order of $S(k)$-in the GPY method. However, not all weight functions are easy to handle, and a Bessel function - even if an analytic function with relatively strongly convergent series expansionmay be unmanageable, at least in our current technical abilities. We will discuss, using the classical series expansion of $J_{m}$, how it may work in this context. Actually, not too well.

With the notations from the above for $q_{r}, \alpha_{m, r}$ etc., (29) yields

$$
q_{r}(x)=x^{-m / 2} \beta^{-m} J_{m}(2 \beta \sqrt{x})=\sum_{j=0}^{\infty} \frac{(-b)^{j}}{j !(m+j) !} x^{j}, \quad \text { with } \quad \beta:=\sqrt{b}, b=\beta^{2}=\frac{\alpha_{m, r}^{2}}{4},
$$

Then we can evaluate the functionals

$G\left(q_{r}\right)=\int_{0}^{1} x^{m+1} q_{r}^{\prime 2}(x) d x, \quad F\left(q_{r}\right)=\int_{0}^{1}(m+1) x^{m} q_{r}^{2}(x) d x=(-2) \int_{0}^{1} x^{m+1} q_{r}(x) q_{r}^{\prime}(x) d x$.

Computations with the power series provide

$$
G\left(q_{r}\right)=b^{2} \sum_{j, \ell=0}^{\infty} \frac{(-b)^{j+\ell}}{(m+j+\ell+2) j ! \ell !(m+j+1) !(m+\ell+1) !},
$$

and

$$
F\left(q_{r}\right)=2 b \sum_{j, \ell=0}^{\infty} \frac{(-b)^{j+\ell}}{(m+j+\ell+2) j ! \ell !(m+j) !(m+\ell+1) !} .
$$

With some reformulations we can also write

$$
\begin{aligned}
& \frac{F\left(q_{r}\right)}{G\left(q_{r}\right)}=\frac{2}{b} \frac{\sum_{\nu=0}^{\infty} \frac{(-b)^{\nu}}{(m+\nu) !(m+\nu+2) !} \sum_{j=0}^{\nu}\left(\begin{array}{c}
m+\nu \\
\nu-j
\end{array}\right)\left(\begin{array}{c}
m+\nu+1 \\
j
\end{array}\right)}{\sum_{\nu=0}^{\infty} \frac{(-b)^{\nu}}{(m+\nu+1) !(m+\nu+2) !} \sum_{j=0}^{\nu}\left(\begin{array}{c}
m+\nu+1 \\
\nu-j
\end{array}\right)\left(\begin{array}{c}
m+\nu+1 \\
j
\end{array}\right)} \\
& =\frac{2}{b} \frac{\sum_{\nu=0}^{\infty} \frac{(-b)^{\nu}}{(m+\nu) !(m+\nu+2) !}\left(\begin{array}{c}
2 m+2 \nu+1 \\
\nu
\end{array}\right)}{\sum_{\nu=0}^{\infty} \frac{(-b)^{\nu}}{(m+\nu+1) !(m+\nu+2) !}\left(\begin{array}{c}
2 m+2 \nu+2 \\
\nu
\end{array}\right)}=\frac{1}{b} \frac{\sum_{\nu=0}^{\infty} \frac{(-b)^{\nu}(2 m+\nu+2)}{(m+\nu+1) !(m+\nu+2) !}\left(\begin{array}{c}
2 m+2 \nu+2 \\
\nu
\end{array}\right)}{\sum_{\nu=0}^{\infty} \frac{(-b)^{\nu}}{(m+\nu+1) !(m+\nu+2) !}\left(\begin{array}{c}
2 m+2 \nu+2 \\
\nu
\end{array}\right)} \\
& =\frac{2}{b} \frac{\sum_{\nu=0}^{\infty} \frac{(-b)^{\nu}}{(2 m+2 \nu+2) !}\left(\begin{array}{c}
2 m+2 \nu+2 \\
m+\nu
\end{array}\right)\left(\begin{array}{c}
2 m+2 \nu+1 \\
\nu
\end{array}\right)}{\sum_{\nu=0}^{\infty} \frac{(-b)^{\nu}}{(2 m+2 \nu+3) !}\left(\begin{array}{c}
2 m+2 \nu+3 \\
m+\nu+1
\end{array}\right)\left(\begin{array}{c}
2 m+2 \nu+2 \\
\nu
\end{array}\right)}=\frac{2}{b} \frac{\sum_{\nu=0}^{\infty} \frac{(2 m+2 \nu+1) !}{(m+\nu) !(m+\nu+2) !(2 m+\nu+1) ! \nu !}(-b)^{\nu}}{\sum_{\nu=0}^{\infty} \frac{(2 m+2 \nu+2) !}{(m+\nu+1) !(m+\nu+2) !(2 m+\nu+2) ! \nu !}(-b)^{\nu}} .
\end{aligned}
$$

Unfortunately the series expansions here have large and oscillating terms, so dealing with it does not seem to be simple. When, e.g., $b$ is of the order $m^{2}$, then also the terms with $\nu \approx m$ are the highest, and there are a large number of similar order large terms. Therefore, this series expansion does not seem to be suitable neither for the computation of the value of the ratio, nor for the extraction of a good polynomial approximation which would approach the global maximum while remaining manageable.

\section{Approximate maximization By polynomials}

5.1. In the aimed applications in showing small gaps between consecutive prime numbers it is very important to have a suitably nice, manageable function $P$. It is enough to mention that even in the simplest case of $P(x)=x^{k+\ell}, \ell \asymp \sqrt{k}$ the technical difficulties become rather serious when $k$ and $\ell$ tend to infinity with the size $N$ of the primes, see [5]. The details of these aspects, when the choice of the weight function is done according 
to the present work, will be handled in the forthcoming paper [8]. Here we will only present the foreseen choice of the weight $P$, and show its approximate optimality. The said choice will be a relatively simple function, actually a real polynomial $P(x)$, satisfying the conditions

$$
x^{k} \mid P(x), P(1)>0, \operatorname{deg} P(x)=k+C_{0} k^{1 / 3},
$$

which is essentially optimal in the extremal problem (1). More exactly, with the notations

$$
A_{k}:=\int_{0}^{1} \frac{x^{k-2}}{(k-2) !}\left(P^{(k-1)}(1-x)\right)^{2} d x, \quad B_{k}:=\int_{0}^{1} \frac{x^{k-1}}{(k-1) !}\left(P^{(k)}(1-x)\right)^{2} d x
$$

it satisfies with an absolute constant $C_{1}$

$$
A_{k}\left(k+C_{1} k^{1 / 3}\right)-4 B_{k} \geq 0
$$

Equivalently,

$$
S(P, k):=\frac{A_{k}}{B_{k}} \geq \frac{4}{k+C_{1} k^{1 / 3}}
$$

in full correspondence with (30). Compared with the optimal transformed Bessel function $q_{1}(x)=x^{-m / 2} J_{m}\left(\alpha_{m, 1} \sqrt{x}\right)$, the difference is only in the value of the constant $C_{1}$.

In order to define our polynomial we put

$$
M:=\left\lceil C_{1} k^{1 / 3} / 6\right\rceil, \quad g(y):=(y-1)^{4}(2-y)^{4} .
$$

Let us remark that the exact choice of $g(y)$ is irrelevant, any positive polynomial or even a function $g \in C^{1}[1,2]$ with a zero of order at least 3 at $y=1$ and $y=2$ would suffice for our purposes. After this, let

$$
P(x):=P_{k}(x):=\sum_{\substack{\ell=M \\ 2 \nmid \ell}}^{2 M} g\left(\frac{\ell}{M}\right)\left(\frac{k}{2}\right)^{\ell} \frac{x^{k+\ell}}{(k+\ell) !} .
$$

5.2. In evaluating $A_{k}$ and $B_{k}$ we will use the well-known relation (easily obtained by partial integration and induction) for the Euler integral

$$
B(m, n):=\int_{0}^{1} x^{n}(1-x)^{m} d x=\frac{m !}{(n+1) \cdots(m+n)} \int_{0}^{1} x^{m+n} d x=\frac{n ! m !}{(m+n+1) !} .
$$


In view of (36) - (37) we have

$$
\begin{aligned}
P^{(k-1)}(x) & =\sum_{\substack{\ell=M \\
2 \nmid \ell}}^{2 M} g\left(\frac{\ell}{M}\right)\left(\frac{k}{2}\right)^{\ell} \frac{x^{\ell+1}}{(\ell+1) !}, \\
P^{(k)}(x) & =\sum_{\substack{\ell=M \\
2 \nmid \ell}}^{2 M} g\left(\frac{\ell}{M}\right)\left(\frac{k}{2}\right)^{\ell} \frac{x^{\ell}}{\ell !}, \\
\left(P^{(k-1)}(x)\right)^{2}= & \sum_{\substack{\ell_{1}=M \\
2 \nmid \ell_{1}}}^{2 M} \sum_{\substack{\ell_{2}=M \\
2 \nmid \ell_{2}}}^{2 M} g\left(\frac{\ell_{1}}{M}\right) g\left(\frac{\ell_{2}}{M}\right)\left(\frac{k}{2}\right)^{\ell_{1}+\ell_{2}} \frac{x^{\ell_{1}+\ell_{2}+2}}{\left(\ell_{1}+1\right) !\left(\ell_{2}+1\right) !}, \\
\left(P^{(k)}(x)\right)^{2}= & \sum_{\substack{\ell_{1}=M \\
2 \nmid \ell_{1}}}^{2 M} \sum_{\substack{\ell_{2}=M \\
2 \nmid \ell_{2}}}^{2 M} g\left(\frac{\ell_{1}}{M}\right) g\left(\frac{\ell_{2}}{M}\right)\left(\frac{k}{2}\right)^{\ell_{1}+\ell_{2}} \frac{x^{\ell_{1}+\ell_{2}}}{\ell_{1} ! \ell_{2} !}, \\
A_{k}= & \sum_{\substack{\ell_{1}=M \\
2 \nmid \ell_{1}}}^{2 M} \sum_{\substack{\ell_{2}=M \\
2 \nmid \ell_{2}}}^{2 M} g\left(\frac{\ell_{1}}{M}\right) g\left(\frac{\ell_{2}}{M}\right) \frac{(k / 2)^{\ell_{1}+\ell_{2}}}{\left(k+\ell_{1}+\ell_{2}+1\right) !}\left(\begin{array}{c}
\ell_{1}+\ell_{2}+2 \\
\ell_{1}+1
\end{array}\right), \\
B_{k}= & \sum_{\substack{\ell_{1}=M \\
2 \nmid \ell_{1}}}^{2 M} \sum_{\substack{\ell_{2}=M \\
2 \nmid \ell_{2}}}^{2 M} g\left(\frac{\ell_{1}}{M}\right) g\left(\frac{\ell_{2}}{M}\right) \frac{(k / 2)^{\ell_{1}+\ell_{2}}}{\left(k+\ell_{1}+\ell_{2}\right) !}\left(\begin{array}{c}
\ell_{1}+\ell_{2} \\
\ell_{1}
\end{array}\right) .
\end{aligned}
$$

In the following, put

$$
u:=\ell_{1}+1, v:=\ell_{2}+1, \quad \text { and } \quad H:=(u+v) / 2, D:=(u-v) / 2 .
$$

Taking into account $2 \nmid \ell_{1}, \ell_{2}$ and $g(1)=g(2)=0$, the even variables $u, v$ will run from $M+1$ to $2 M$ and we will have

$$
\frac{k^{\ell_{1}+\ell_{2}} k !}{\left(k+\ell_{1}+\ell_{2}\right) !}=1+O\left(\frac{M^{2}}{k}\right)
$$

Here and elsewhere in the sequel the implied absolute constants of the $O$ symbol as well as the absolute constants $C_{i}(i \geq 2)$ will be always independent from $C_{1}$.

Clearly, we can replace $A_{k}^{\prime}:=k ! A_{k}$ and $B_{k}^{\prime}:=k ! B_{K}$ for $A_{k}$ and $B_{k}$, resp., in (33), hence in order to show (33) it suffices to prove

$$
\sum_{H=M+1}^{2 M} I_{k}(H)\left(1+O\left(\frac{M^{2}}{k}\right)\right) \geq 0
$$

where

$$
I_{k}(H):=2^{-2 H} \sum_{\substack{2 \mid u, v, u+v=2 H \\
u, v \in(M, 2 M]}} g\left(\frac{u-1}{M}\right) g\left(\frac{v-1}{M}\right)\left(\begin{array}{c}
u+v-2 \\
u-1
\end{array}\right)\left\{\frac{k+C_{1} k^{1 / 3}}{k+4 M} \frac{(u+v)(u+v-1)}{u v}-4\right\} .
$$


Let us denote the above summation conditions simply by $\sum_{u, v}^{*}$ and let us consider first

$$
\begin{aligned}
I_{k}^{\prime}(H) & :=2^{-2 H} \sum_{u, v}^{*} g\left(\frac{u-1}{M}\right) g\left(\frac{v-1}{M}\right)\left(\begin{array}{c}
u+v-2 \\
u-1
\end{array}\right)\left\{\frac{(u+v)(u+v-1)}{u v}-4\right\} \\
48) \quad & =2^{-2 H} \sum_{u, v}^{*} g\left(\frac{u-1}{M}\right) g\left(\frac{v-1}{M}\right)\left(\begin{array}{c}
u+v-2 \\
u-1
\end{array}\right) \frac{4 D^{2}-2 H}{H^{2}-D^{2}} .
\end{aligned}
$$

We will see later that the mere significance of the weight function $g$ is to cut the tails when $H$ is near to $M$ or $2 M$, so first we will investigate the simpler sum

$$
I_{k}^{\prime \prime}(H):=2^{-2 H} \sum_{u, v}^{*}\left(\begin{array}{c}
u+v-2 \\
u-1
\end{array}\right) \frac{4 D^{2}-2 H}{H^{2}-D^{2}},
$$

when

$$
H \in[M+t(M), 2 M-t(M)], \quad t(M):=4 \sqrt{M \log M} .
$$

By Stirling's formula

$$
\log \Gamma(s)=\left(s-\frac{1}{2}\right) \log s-s+\frac{1}{2} \log (2 \pi)+O\left(\frac{1}{|s|}\right),
$$

we obtain

$$
\begin{aligned}
\log \left(2^{-2 H}\left(\begin{array}{c}
u+v-2 \\
u-1
\end{array}\right)\right)=\log \left(\frac{\Gamma(2 H-1) 2^{-2 H}}{\Gamma(H+D) \Gamma(H-D)}\right) \\
=\left(2 H-\frac{3}{2}\right)\left(\log H+\log 2+\log \left(1-\frac{1}{2 H}\right)\right)+1-\frac{\log 2 \pi}{2}-2 H \log 2+O\left(\frac{1}{H}\right) \\
\quad-\left(H+D-\frac{1}{2}\right)\left(\log H+\log \left(1+\frac{D}{H}\right)\right)-\left(H-D-\frac{1}{2}\right)\left(\log H+\log \left(1-\frac{D}{H}\right)\right) \\
=\frac{-\log H}{2}-\frac{3 \log 2}{2}-1+1-\frac{\log 2 \pi}{2} \\
\quad-\sum_{n=0}^{\infty}\left(\frac{2 D}{2 n+1}\left(\frac{D}{H}\right)^{2 n+1}-\frac{2 H}{2 n+2}\left(\frac{D}{H}\right)^{2 n+2}\right)+O\left(\frac{1}{H}+\frac{D^{2}}{H^{2}}\right) .
\end{aligned}
$$

$$
=\frac{-\log H}{2}-\frac{\log 16 \pi}{2}-\sum_{n=0}^{\infty} \frac{1}{(n+1)(2 n+1)} \frac{D^{2 n+2}}{H^{2 n+1}}+O\left(\frac{1}{H}+\frac{D^{2}}{H^{2}}\right) .
$$

Using $e^{-|x|}=1+O(x)$ we obtain from (49), (150) and (52)

$$
\begin{aligned}
I_{k}^{\prime \prime}(H) & =\frac{1}{2 \sqrt{\pi} H^{3 / 2}} \sum_{\substack{2 \mid D-H \\
M<H-D, H+D \leq 2 M}} e^{-D^{2} / H}\left(2 \frac{D^{2}}{H}-1\right)\left(1+O\left(\frac{1}{H}+\frac{D^{2}}{H^{2}}+\frac{D^{4}}{H^{3}}\right)\right) \\
& =\frac{I_{k}^{*}(H)}{2 \sqrt{\pi} H^{3 / 2}}+O\left(\frac{1}{M^{2}}\right)+O\left(\int_{t(M)}^{\infty} \frac{t^{2}}{H} e^{-t^{2} / H} d t\right)=\frac{I_{k}^{*}(H)}{2 \sqrt{\pi} H^{3 / 2}}+O\left(\frac{1}{M^{2}}\right),
\end{aligned}
$$

where

$$
I_{k}^{*}(H):=\sum_{\substack{2 \mid m-H \\ m=-\infty}}^{\infty} f(m), \quad f(x)=\left(2 \frac{x^{2}}{H}-1\right) e^{-x^{2} / H}
$$


5.3. We will use Poisson summation formula

$$
\sum_{n=-\infty}^{\infty} f(t+n T)=\frac{1}{T} \sum_{\nu=-\infty}^{\infty} \widehat{f}\left(\frac{\nu}{T}\right) e^{2 \pi i \nu t / T}
$$

with $T=2, t=0$ for $2 \mid H$ and $T=2, t=1$ for $2 \nmid H$, valid ift

$$
|f(x)|+|\widehat{f}(x)| \leq C\left(1+|x|^{-1-\delta}\right) \quad \text { with some } \quad \delta>0, C>0 .
$$

Let us consider first the case when $H$ is even, that is when $m=2 n$ in the above sum (54). We have then

$$
\begin{aligned}
& 2 I_{k}^{*}(H)=2 \sum_{n=-\infty}^{\infty} f(2 n)=\sum_{\nu=-\infty}^{\infty} \widehat{f}(\nu / 2)=\sum_{\nu=-\infty}^{\infty} \int_{-\infty}^{\infty} f(x) e^{\pi i \nu x} d x \\
& =\sum_{\nu=-\infty}^{\infty} \int_{-\infty}^{\infty} e^{-x^{2} / H}\left(2 \frac{x^{2}}{H}-1\right) e^{\pi i \nu x} d x=\sqrt{H} \sum_{\nu=-\infty}^{\infty} \int_{-\infty}^{\infty} e^{-y^{2}}\left(2 y^{2}-1\right) e^{\pi i \nu \sqrt{H} y} d y \\
& =\sqrt{H} \sum_{\nu=-\infty}^{\infty} \int_{\Im y=0} e^{-(y-\pi i \nu \sqrt{H} / 2)^{2}-\pi^{2} \nu^{2} H / 4} \\
& \cdot\left\{2(y-\pi i \nu \sqrt{H} / 2)^{2}+2(y-\pi i \nu \sqrt{H} / 2) \pi i \nu \sqrt{H}-\pi^{2} \nu^{2} H / 2-1\right\} d y \\
& =\sqrt{H} \sum_{\nu=-\infty}^{\infty} \int_{\Im z=-\pi \nu \sqrt{H} / 2} e^{-z^{2}-\pi^{2} \nu^{2} H / 4}\left\{2 z^{2}+2 \pi i \nu \sqrt{H} z-\pi^{2} \nu^{2} H / 2-1\right\} d z \\
& =\sqrt{H} \sum_{\nu=-\infty}^{\infty} \int_{\Im z=0} e^{-z^{2}-\pi^{2} \nu^{2} H / 4}\left\{2 z^{2}+2 \pi i \nu \sqrt{H} z-\pi^{2} \nu^{2} H / 2-1\right\} d z \\
& =\sqrt{H} \int_{z \in \mathbb{R}} e^{-z^{2}}\left(2 z^{2}-1\right) d z+O\left(e^{-2 H}\right)=\sqrt{H}\left[-z e^{-z^{2}}\right]_{-\infty}^{\infty}+O\left(e^{-2 H}\right)=O\left(e^{-2 H}\right) .
\end{aligned}
$$

The proof runs completely analogously for $t=1$, that is, when $H$ is odd, because the extra factor $e^{\pi i \nu}$ does not change the modulus of $\widehat{f}(\nu / 2)$.

5.4. Dealing with the original integral $I_{k}^{\prime}(H)$ we have to take into account the effect of the weight function $g$ as well. From the Taylor expansion of $g$ we find

$$
g\left(\frac{H \pm D-1}{M}\right)=g\left(\frac{H-1}{M}\right) \pm g^{\prime}\left(\frac{H-1}{M}\right) \frac{D}{M}+O\left(\frac{D^{2}}{M^{2}}\right)
$$

so this effect is

$$
g\left(\frac{H+D-1}{M}\right) g\left(\frac{H-D-1}{M}\right)=g^{2}\left(\frac{H-1}{M}\right)+O\left(\frac{D^{2}}{M^{2}}\right) .
$$

The effect of the error term on $I_{k}^{\prime}(H)$ is here, similarly to (49),

$$
O\left(H^{-3 / 2} \sum_{\substack{2|D-H\\| D \mid<M}} e^{-D^{2} / H}\left(1+\frac{D^{2}}{H}\right)\left(1+\frac{D^{4}}{H^{3}}\right) \frac{D^{2}}{M^{2}}\right)=O\left(\frac{1}{M^{2}}\right) .
$$

\footnotetext{
${ }^{4}$ For this form see [12, Chapter VII, §2].
} 
So we obtain for all integers $H$ subject to (50) from (48), (49), (53), (55), (57), (59) and (60)

$$
I_{k}^{\prime}(H)=I_{k}^{\prime \prime}(H) g^{2}\left(\frac{H-1}{M}\right)+O\left(\frac{1}{M^{2}}\right)=\frac{g^{2}\left(\frac{H-1}{M}\right)}{2 \sqrt{\pi} H^{3 / 2}} I_{k}^{*}(H)+O\left(\frac{1}{M^{2}}\right) \geq-\frac{C_{2}}{M^{2}} .
$$

On the other hand, if (50) does not hold, that is if

$$
\min (H-M, 2 M-H)<t(M),
$$

then we find $|D|<t(M)$ and $|H \pm D-1| \in[M, M+2 t(M)] \cup[2 M-2 t(M), 2 M]$, whence

$$
g\left(\frac{H+D-1}{M}\right) g\left(\frac{H-D-1}{M}\right) \ll \frac{t^{4}(M)}{M^{4}} \ll \frac{1}{M^{7 / 4}} .
$$

This implies in case of (62)

$$
I_{k}^{\prime}(H) \ll M^{-7 / 4} M^{-3 / 2} \int_{-\infty}^{\infty} e^{-u^{2} / H}\left(\frac{u^{2}}{H}+1\right)^{3} d u \ll M^{-11 / 4} .
$$

Consequently, for $k>k_{0}$ we have by $I_{k}(H) \geq I_{k}^{\prime}(H)$

$$
\sum_{\substack{H \\ \min (H-M, 2 M-H)<t(M)}} I_{k}(H) \geq \sum_{\substack{H \\ \min (H-M, 2 M-H)<t(M)}} I_{k}^{\prime}(H) \geq-C_{3} M^{-2} .
$$

Finally, let us investigate now for $H$ in (50) the difference

$$
I_{k}(H)-I_{k}^{\prime}(H) \geq \frac{M}{k} \cdot 3 \widetilde{I}_{k}(H),
$$

where similarly to the above considerations

$$
\begin{aligned}
\widetilde{I}_{k}(H):=2^{-2 H} \sum_{u, v}^{*} g\left(\frac{u-1}{M}\right) g\left(\frac{v-1}{M}\right)\left(\begin{array}{c}
u+v-2 \\
u-1
\end{array}\right) \\
=\frac{1}{2 \sqrt{\pi H}} \sum_{\substack{2 \mid D-H \\
M<H-D, H+D \leq 2 M}} e^{-D^{2} / H}\left(1+O\left(\frac{1}{H}+\frac{D^{2}}{H^{2}}+\frac{D^{4}}{H^{3}}\right)\right) \\
\cdot\left(g^{2}\left(\frac{H-1}{M}\right)+O\left(\frac{D^{2}}{M^{2}}\right)\right) \geq C_{4} g^{2}\left(\frac{H-1}{M}\right)-\frac{C_{5}}{M} .
\end{aligned}
$$

Summing over all $H$ in (62) we obtain

$$
\sum_{H=M+t(M)}^{2 M-t(M)} I_{k}(H) \geq \sum_{H=M+t(M)}^{2 M-t(M)} I_{k}^{\prime}(H)+\frac{C_{6} M^{2}}{k} .
$$

Therefore by (61) and (65)-(68) we have finally

$$
\sum_{H=M+1}^{2 M} I_{k}(H) \geq \frac{C_{6} M^{2}}{k}-\frac{C_{2}}{M}-\frac{C_{3}}{M^{2}}>0,
$$

if $C_{1}$ was chosen sufficiently large, satisfying

$$
C_{1}>6\left(\frac{C_{2}}{C_{6}}\right)^{1 / 3}
$$




\section{REFERENCES}

[1] M. Abramowitz and I. A. Stegun, Handbook of mathematical functions with formulas, graphs, and mathematical tables, National Bureau of Standards Applied Mathematics Series 55, For sale by the Superintendent of Documents, U.S. Government Printing Office, Washington, D.C., 1964.

[2] F. Bowman, Introduction to Bessel functions, Dover Publications Inc., New York, 1958.

[3] J. Friedlander and H. Iwaniec, Opera de cribro, American Mathematical Society Colloquium Publications 57, American Mathematical Society, Providence, RI, 2010.

[4] D. A. Goldston, J. Pintz and C. Y. Yıldırım, Primes in tuples. I, Ann. of Math. (2) 170 (2009), 819-862.

[5] _ Primes in tuples. II, Acta Math. 204 (2010), 1-47.

[6] D. A. Goldston, Y. Motohashi, J. Pintz and C. Y. Ylldir im, Small gaps between primes exist, Proc. Japan Acad. Ser. A Math. Sci. 82 (2006), 61-65.

[7] P. Meyer-Nieberg, Banach lattices, Universitext, Springer-Verlag, Berlin, 1991.

[8] J. Pintz, Some new results on gaps between consecutive primes, in: Paul Turán Memorial Volume: Number Theory, Analysis and Combinatorics, 2013, de Gruyter, Berlin, to appear.

[9] F. Riesz and B. Sz.-Nagy, Functional analysis, Dover Books on Advanced Mathematics, Dover Publications Inc., New York, 1990, Translated from the second French edition by Leo F. Boron, Reprint of the 1955 original.

[10] K. Soundararajan, Note on Goldston-Pintz-Yıldırım, (an analysis of the GPY result), (2005), unpublished note, sent in an email letter to D. Goldston.

[11] Small gaps between prime numbers: the work of Goldston-Pintz-Yıldırım, Bull. Amer. Math. Soc. (N.S.) 44 (2007), 1-18.

[12] E. M. Stein and G. Weiss, Introduction to Fourier analysis on Euclidean spaces, Princeton University Press, Princeton, N.J., 1971, Princeton Mathematical Series, No. 32.

Bergische Universität Wuppertal

Faculty of Mathematics and Natural Science

Gaussstrasse 20, 42119 Wuppertal, GERMANY

E-mail address: farkas@uni-wuppertal.de

Alfréd RÉnyi Institute of Mathematics

Hungarian ACADEMY OF SCIENCES

1364 BUDAPEST, PO Box 127, HUNGARY

E-mail address: pintz@renyi.mta.hu

revesz.szilard@renyi.mta.hu

Department of Mathematics

KuWAit University

P.O. Box 5969 SAFAT - 13060 KUWAIT

E-mail address: szilard@sci.kuniv.edu.kw 